\title{
Do All Prostate Cancers Behave the Same?
}

\author{
Dissanayake Thusitha $^{{ }^{*}}$, Arze Elizabeth ${ }^{2}$, Rogers Mailien ${ }^{3}$ \\ ${ }^{1}$ Department of Internal Medicine, James H. Quillen College of Medicine, East Tennessee State University, Johnson City, USA; \\ ${ }^{2}$ Department of Pathology, James H. Quillen College of Medicine, East Tennessee State University, Johnson City, USA; ${ }^{3}$ Division of \\ Hematology-Oncology, James H. Quillen College of Medicine, East Tennessee State University, Johnson City, USA. \\ Email: *thusithard007@gmail.com
}

Received August 27 $7^{\text {th }}$, 2013; revised September 25 $5^{\text {th }}$, 2013; accepted October $3^{\text {rd }}$, 2013

Copyright (C) 2013 Dissanayake Thusitha et al. This is an open access article distributed under the Creative Commons Attribution License, which permits unrestricted use, distribution, and reproduction in any medium, provided the original work is properly cited.

\begin{abstract}
Two different immunohistochemical types suggestive of Large Cell Neuroendocrine (NE) carcinoma and Adenocarcinoma in a patient with known diffusely metastatic, hormone refractory prostate carcinoma are rarities. Interestingly, our patient had documented history of exposure to Agent Orange during his time of service. The use of routinely used immunohistochemical stains for pathological diagnosis was a challenge in this case, though throughout his disease course, the diagnosis was confirmed as Adenocarcinoma of prostate with biopsies from all various sites of metastases. Systemic chemotherapy has been historically suboptimal in management of aggressively behaved prostate carcinomas. Finding any association of Agent Orange as a causative etiology and improving diagnosis and management of such aggressive hormone refractory prostate carcinoma need further investigations.
\end{abstract}

Keywords: Large Cell Neuroendocrine Carcinoma; Adenocarcinoma of Prostate; Hormone Refractory Prostate Carcinoma; Agent Orange

\section{Introduction}

A 62-year-old white male was diagnosed with metastatic hormone refractory adenocarcinoma of prostate and was treated with luteinizing hormone releasing hormone agonist. The patient gave a history of melanoma and remote history of tobacco abuse. Several months after treatment was initiated, the patient was presented to the emergency room for stroke like symptoms and was found to have brain metastases secondary to his prostate.

Further workup revealed a mediastinal lymph node with inconclusive biopsy result for a primary though adenocarcinoma was primarily suspected.

We present an interesting yet complicated case raising the question of prostate cancer with exposure to Agent Orange, to which our patient was exposed during the Vietnam War.

\section{Case Report and Discussion}

A Vietnam veteran was originally diagnosed with Stage IV Prostate Adenocarcinoma July 2011 when he presented with new onset urinary retention.

He underwent cystoscopy July 26th, 2011 and 4 out of

"Corresponding author.
4 core biopsies were reported as adenocarcinoma of prostate with Gleason score of 8.

His bone scan showed extensive bony metastases.

At the time of diagnosis, his PSA was only mildly elevated at 5.66. He was started on androgen deprivation therapy (ADT) with Zoladex injections.

In November 2011, patient presented to VA ER with vomiting and left sided weakness. On suspicion of cerebral vascular accident, CT scanning revealed a $4-\mathrm{cm}$ right frontal white matter centrally necrotic mass with surrounding vasogenic edema. He was transferred to local level 4 tertiary care center and brain biopsy performed.

Due to rarity of adenocarcinoma of prostate metastasizing to the brain, search for more common sources were sought. Further investigation proved his history of melanoma was actually squamous cell but due to history of smoking, lung primary was also a possibility.

Pathology of brain biopsies reported as tumor cells showing sheets of neoplastic cells with prominent gland and cruciform formation with marked nuclear atypia with easily visible mitosis. The morphological features were consistent with prostate adenocarcinoma per pathology report (Figures 1(a) and (b)).

The submitted PSA immunohistochemical stains at the outside facility were negative which is common after 


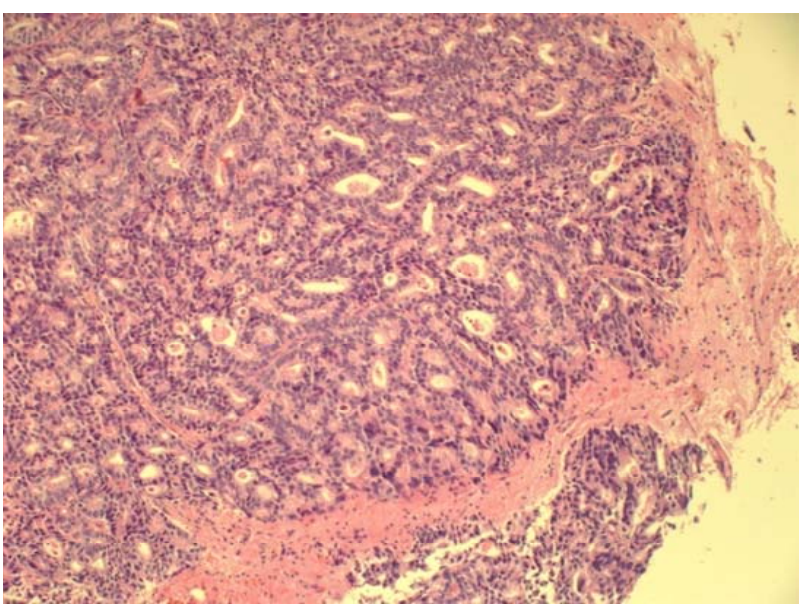

(a)

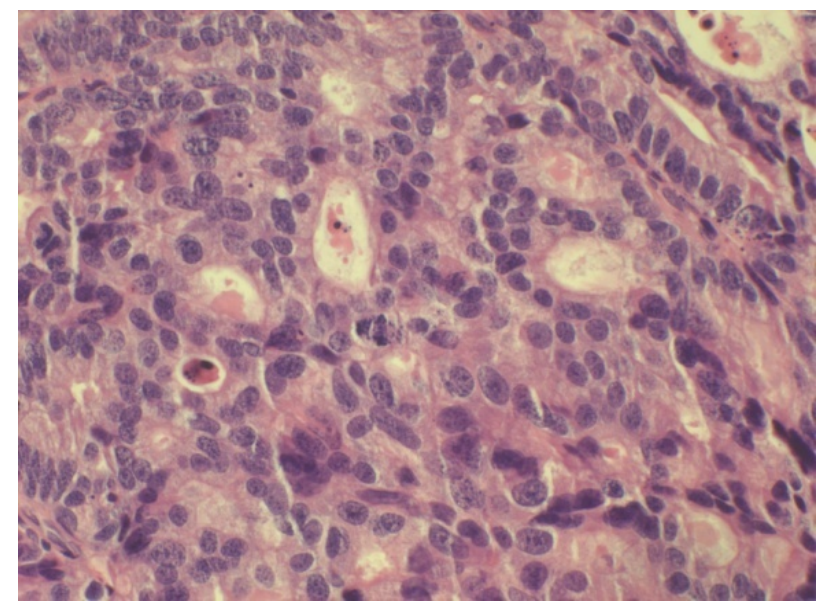

(b)

Figure 1. (a) Bran biopsy in low power; (b) Brain biopsy in hi power. The tumor cells show sheets of neoplastic cells with prominent gland and cribriform formation with marked nuclear atypia. Mitosis is easily visible. The morphologic features are consistent with prostatic adenocarcinoma, however, the submitted PSA immunohisto-chemical stain performed at the outside facility is negative.

initiation of ADT. The rest of the submitted slides showed that the tumor cells were strongly reactive for CK7 and negative for CK20 and TTF1. The negative TTF1 made lung and thyroid origin less likely. The outside institution also performed HMB, MART-1 and G-FAP immunohistochemical stains. Per report the tumor cells are negative for HMB and MART-1 which exclude melanoma. The tumor cells are also negative for GFAP which excludes an ependymal and astrocytic lesion [1].

His oncology care was transferred to VA Hematology/ Oncology department in November 2011. Colonoscopy was performed and revealed negative results so his androgen deprivation therapy was continued through his disease course. Follow up imaging with MRI spine showed more bony disease so PET/CT was performed to exclude new primary.

PET/CT only avid in subcarinal area so endobronchial ultrasound guided biopsy (EBUS) was performed and again pathological diagnosis of adenocarcinoma; likely, prostate as the primary site. Immunohistochemistry reported strongly positive for CK7, focally positive for CK20, but negative for TTF-1, p63 and PSA.

The patient was started on Docetaxol and prednisone and had a total of 6 cycles. His treatment was complicated with new neurological symptoms but imaging only showed increased edema around his previous intracranial lesion treated with local radiation; cyber knife therapy.

In mid October 2012, assessment which included CT of Chest with contrast revealed new extensive hepatic metastasis with the largest lesion measuring of about 7.6 $\times 7.4 \mathrm{~cm}$ which was biopsied under CT guidance. The pathology reported Immunoperoxidase studies showing the moderate reactivity of tumor cells with AE1/AE3, moderate reactivity with chromogranin, moderate to strong reactivity with synaptophysin and negative reactivity with CD56 (Figures 2 and 3).

Tumor cells also showed moderate to marked reactivity with TTF-1, but are negative for CK7, CK20, PSA and CD45. Tumor cells also show mild to moderate reactivity with S100 protein. There were no small cells present [1].

These immunophenotypic characteristics were consistent with TTF-1 positive large neuroendocrine (NE) carcinoma which comprises the small subset of non-small cell carcinomas. Per pathology report, there is a rare possibility of large cell neuroendocrine carcinomas with TTF-1 positivity of genitourinary origin [2,3].

Biopsy slides of subcarinal lymph nodes were again looked at carefully and revealed a very small population of TTF-1 positive cells (though this was not officially documented anywhere). It appeared there were 2 distinct clones in his subcarinal metastatic lesions [4].

Within one week of discovery of his hepatic lesions, he fell very ill and could not be started on any further chemotherapy and succumbed to his advanced illness.

\section{Conclusions}

Management of hormone refractory prostate cancer has been always a challenge in therapy and follow-up for prognosis. When metastasis occurs, there will be a point that response to androgen deprivation therapy will decline until one becomes hormone refractory.

Systemic chemotherapy has been historically suboptimal in regard to prolonged responses and tolerance. Markers like PSA have become less useful in this situation.

Two different immunohistochemical types of cells from the same biopsy in a veteran with a history of ex- 


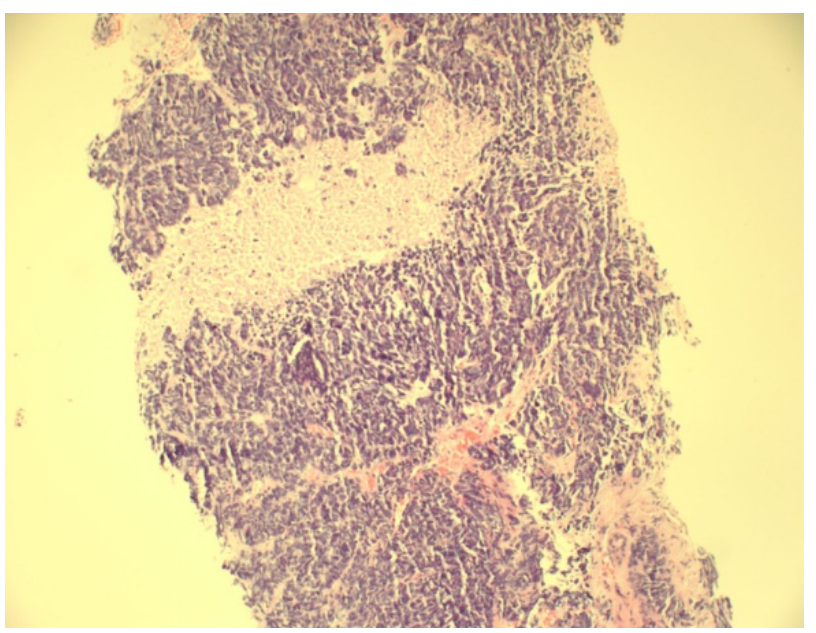

(a)

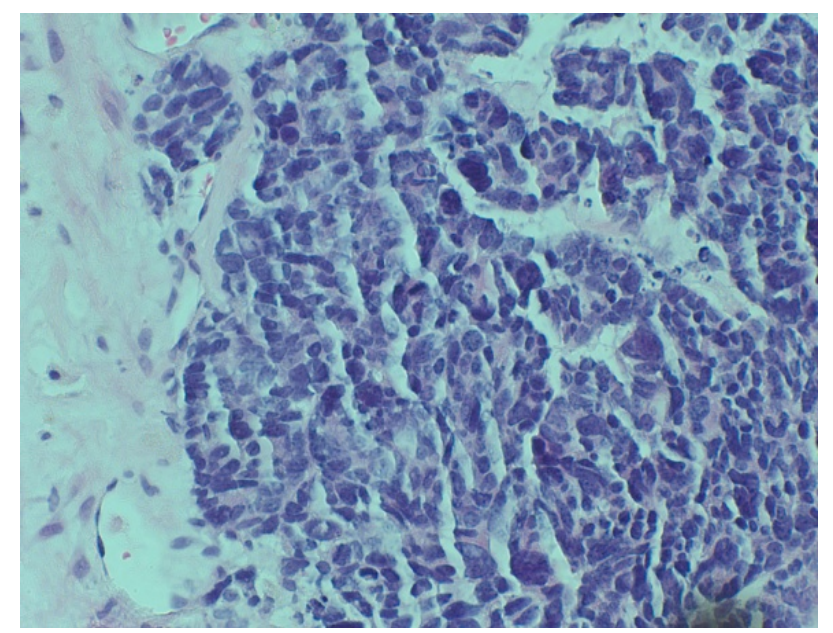

(b)

Figure 2. (a) Liver biopsy in low power; (b) Liver biopsy in high power. Sections show fragments of fibrovasculer tissue infiltrated by a poorly differentiated carcinoma with zonal coagulation necrosis and moderate desmoplastic reaction. Tumor cells have variable large nucleoli with coarse chromatin, occasional large nucleoli and scant cytoplasm. Mitotic activity is high, including atypical mitosis and scattered tumor giant cells.

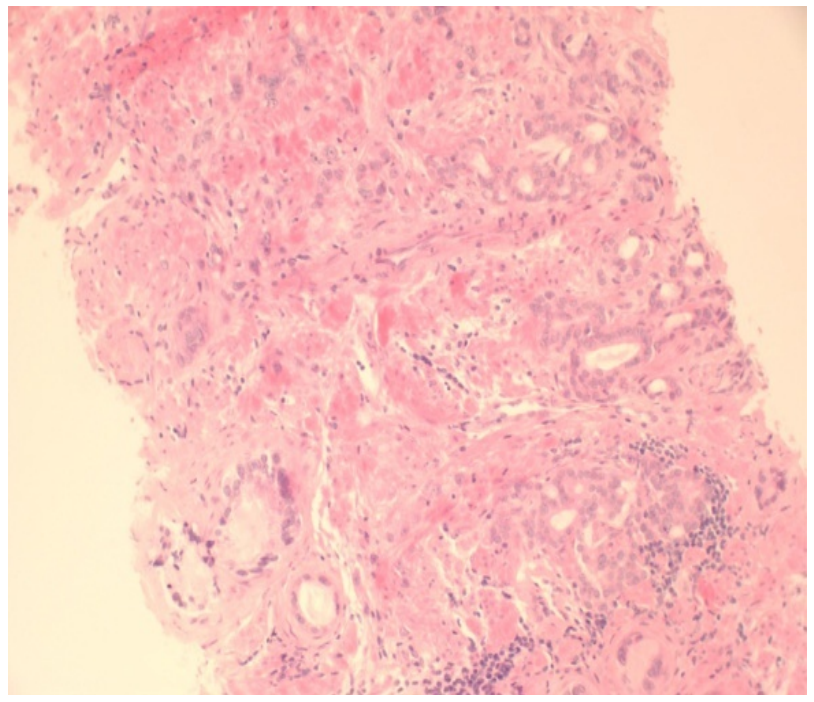

Figure 3. Prostate biopsy. Four out of four core biopsy specimens contain prostatic adenocarcinoma with a predominant Gleason Grade of $4+4=8$. There is also presented a focal area with Gleason Grade 5 adenocarcinoma.

posure to Agent Orange are an unusual presentation.

As with our patient, the aggressiveness of the disease and use of commonly available immunohistochemical stains for pathological diagnosis can be a challenge.

Finding an association of Agent Orange as a causative etiology, and improving diagnosis, and management of such aggressive hormone refractory prostate carcinoma need further research.

\section{REFERENCES}

[1] O. Kaufmann and M. Dietel, "Expression of Thyroid Transcription Factor-1 in Pulmonary and Extrapulmonary Small Cell Carcinomas and Other Neuroendocrine Carcinomas of Various Primary Sites,” Histopathology, Vol. 36, No. 5, 2000, pp. 415-420. http://dx.doi.org/10.1046/j.1365-2559.2000.00890.x

[2] R. Mazzucchelli, D. Morichetti, A. Lopez-Beltran, L. Cheng, M. Scarpelli, Z. Kirkali and R. Montironi, "Neuroendocrine Tumours of the Urinary System and Male Genital Organs: Clinical Significance,” BJU International, Vol. 103, No. 11, 2009, pp. 1465-1470. http://dx.doi.org/10.1111/j.1464-410X.2009.08451.x

[3] K. Hiroshima, A. Iyoda, T. Shida, K. Shibuya, T. Iizasa, H. Kishi, T. Tanizawa, T. Fujisawa and Y. Nakatani, "Distinction of Pulmonary Large Cell Neuroendocrine Carcinoma from Small Cell Lung Carcinoma: A Morphological, Immunohistochemical, and Molecular Analysis,” Modern Pathology, Vol. 19, No. 10, 2006, pp. 13581368. http://dx.doi.org/10.1038/modpathol.3800659

[4] A. J. Evans, P. A. Humphrey, J. Belani, T. H. van der Kwast and J. R. Srigley, "Large Cell Neuroendocrine Carcinoma of Prostate: A Clinicopathologic Summary of 7 Cases of a Rare Manifestation of Advanced Prostate Cancer," American Journal of Surgical Pathology, Vol. 30, No. 6, 2006, pp. 684-693. http://dx.doi.org/10.1097/00000478-200606000-00003 